\title{
Impact of the plasma geometry on divertor power exhaust: experimental evidence from TCV and simulations with SolEdge2D and TOKAM3X
}

\author{
A Gallo ${ }^{1}(1)$, N Fedorczak ${ }^{1}$, S Elmore ${ }^{2}$, R Maurizio $^{3}$, H Reimerdes $^{3}(1)$,

 \\ G Ciraolo ${ }^{1}$, D Galassi ${ }^{6}$, P Ghendrih ${ }^{1}$, M Valentinuzzi ${ }^{1}$, P Tamain $^{1}$, \\ the EUROfusion MST1 team ${ }^{7}$ and the TCV team ${ }^{8}$ \\ ${ }^{1}$ IRFM, CEA-Cadarache, F-13108 Saint-Paul-lez-Durance, France \\ ${ }^{2}$ CCFE, Culham Science Center, Abingdon, OX14 3DB, United Kingdom \\ ${ }^{3}$ Swiss Plasma Center (SPC), Ecole Polytechnique Fédérale de Lausanne (EPFL), CH-1015 Lausanne, \\ Switzerland \\ ${ }^{4}$ University of California-San Diego, La Jolla, CA 92093, United States of America \\ ${ }^{5}$ Max-Planck-Institute for Plasma Physics, Boltzmannstr. 2, D-85748 Garching, Germany \\ ${ }^{6}$ Aix Marseille Univ, CNRS, Centrale Marseille, M2P2, Marseille, France \\ E-mail: alberto.gallo@cea.fr
}

\begin{abstract}
A deep understanding of plasma transport at the edge of magnetically confined fusion plasmas is needed for the handling and control of heat loads on the machine first wall. Experimental observations collected on a number of tokamaks over the last three decades taught us that heat flux profiles at the divertor targets of X-point configurations can be parametrized by using two scale lengths for the scrape-off layer (SOL) transport, separately characterizing the main SOL $\left(\lambda_{q}\right)$ and the divertor SOL $\left(S_{q}\right)$. In this work we challenge the current interpretation of these two scale lengths as well as their dependence on plasma parameters by studying the effect of divertor geometry modifications on heat exhaust in the Tokamak à Configuration Variable. In particular, a significant broadening of the heat flux profiles at the outer divertor target is diagnosed while increasing the length of the outer divertor leg in lower single null, Ohmic, L-mode discharges. Efforts to reproduce this experimental finding with both diffusive (SolEdge2D-EIRENE) and turbulent (TOKAM3X) modelling tools confirm the validity of a diffusive approach for simulating heat flux profiles in more traditional, short leg, configurations while highlighting the need of a turbulent description for modified, long leg, ones in which strongly asymmetric divertor perpendicular transport develops.
\end{abstract}

Keywords: scrape-off layer transport, divertor magnetic geometry, heat load characterization (Some figures may appear in colour only in the online journal)

\section{Introduction}

\subsection{Heat flux limit on plasma facing components}

7 See the author list of H Meyer et al 2017 Nucl. Fusion 57102014.

The successful and safe operation of future magnetic con8 See the author list of S Coda et al 2017 Nucl. Fusion 57102011. finement nuclear fusion reactors like ITER strongly depends 
on overcoming several currently open issues in the fields of physics and technology. An efficient and controlled handling of the power exhaust is one of them. In diverted configurations, during the steady state, part of the power supplied to the confined plasma by the heating systems is transferred to the scrape-off layer (SOL): here unconfined plasma flows towards the vacuum vessel walls both along and transversely to open magnetic field lines. Most of the exhausted heat and particle flux is concentrated onto dedicated components, called divertor targets. Such components, which in ITER will be made out of tungsten, can tolerate a heat flux normal to the material surface up to $q_{\text {surf }}=10 \mathrm{MW} \mathrm{m}^{-2}$ in steady state and of $20 \mathrm{MW} \mathrm{m}^{-2}$ during slow transients [1]. These limits refer to pristine, undamaged, tungsten and might have to be corrected when taking into account the effect of plasma fluence and modifications of the divertor design, as recently shown in [2-4]. It should be noted that, in order to limit $q_{\text {surf }}$, ITER will have to be operated in partially detached conditions [5]. Detached plasmas are beyond the scope of this work, which focuses entirely on attached conditions. A detailed study of the effect of plasma geometry on detachment can be found in [6]. For a given power $P_{\text {div }}$ flowing towards the outer divertor target, the maximum heat flux is determined by the wetted area: $P_{\text {div }}=A_{\text {wet }} q_{\text {surf }}^{\max } \simeq 2 \pi R_{\mathrm{t}} \lambda_{\text {wet }} q_{\text {surf }}^{\max }$, where $R_{\mathrm{t}}$ is the position of the target along the tokamak major radius and $\lambda_{\text {wet }}$ is the wetted width, equivalent of $A_{\text {wet }}$ once toroidal symmetry is assumed. Such quantity depends on the width of the SOL which in turn is determined by plasma transport in the boundary region.

\subsection{Width of the $S O L$}

When talking about the width of the SOL we refer to the distance from the magnetic separatrix over which plasma quantities such as density $n$, temperature $T$ and heat flux $q$ are spread on open magnetic field lines. Such width can be understood as a result of the competition between transport parallel $(\|)$ and perpendicular $(\perp)$ to the total magnetic field $\vec{B}$ [7]. In a particle description, when the plasma reaches the divertor targets, it has spent as much time travelling in the $\|$ as in the $\perp$ direction: $\tau_{\|}=\tau_{\perp}$. This can be rephrased, in terms of distances and velocities, as $L_{\|} / v_{\|}=\lambda_{\mathrm{SOL}} / v_{\perp}$, where $L_{\|}$is the parallel connection length (length of field lines from the outer midplane to the target), $\lambda_{\mathrm{SOL}}$ is the SOL width, $v_{\|}$and $v_{\perp}$ are the velocities in the $\|$ and $\perp$ direction respectively. Since plasma transport is much quicker along field lines than transversely because of confinement, $\lambda_{\mathrm{SOL}}$ is expected to be much smaller than $L_{\|}$. A simplified picture of SOL transport can be obtained assuming that plasma travels at sound speed $c_{\mathrm{s}}$ along field lines while diffusing in the $\perp$ direction: $\lambda_{\mathrm{SOL}}=\sqrt{D_{\perp} L_{\|} / c_{\mathrm{s}}}$, where $D_{\perp}\left[\mathrm{m}^{2} \mathrm{~s}^{-1}\right]$ is the particle diffusivity in the $\perp$ direction. A more sophisticated description would require an expression for $v_{\perp}$ based on, for instance, turbulent arguments. The notation used so far was intentionally generic since $n, T$ and $q$ might be transported at different speed and therefore their profiles could be characterized by different widths. Although the main focus of this work will be on the heat flux width $\lambda_{q}$, its link with the density and temperature

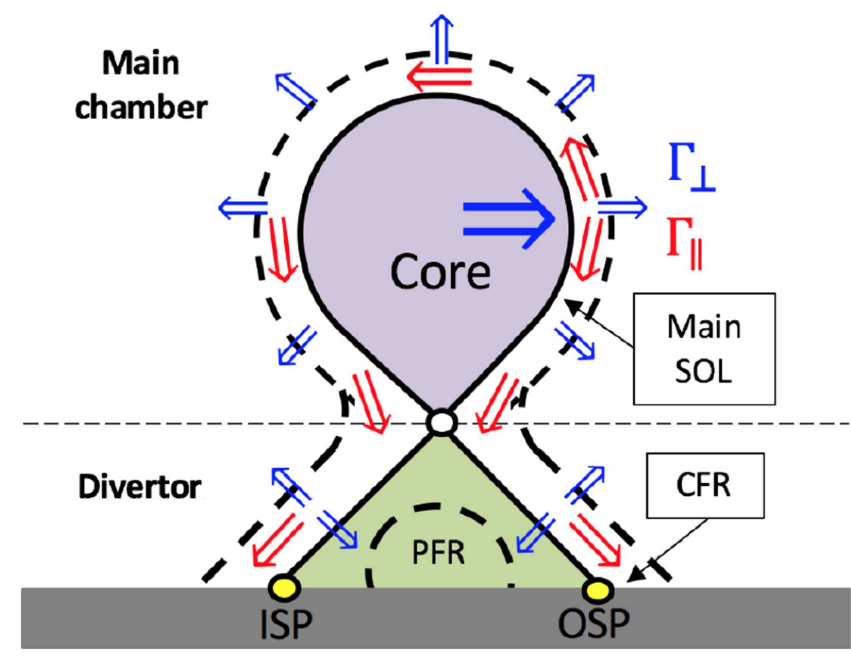

Figure 1. Sketch of a diverted configuration showing plasma core (purple), separatrix (black solid line), a generic SOL $\psi$ surface (thick black dashed line), main SOL (white), CFR (white), PFR (green), divertor target (grey), inner and outer strike points (ISP and OSP, yellow), plasma fluxes parallel and perpendicular to magnetic $\psi$ surfaces (red and blue arrows).

widths, $\lambda_{n}$ and $\lambda_{\mathrm{T}}$ respectively, are important matter for discussion.

\subsection{Parametrization of SOL profiles}

In order to assess their widths, one has to efficiently parametrize radial profiles of SOL quantities. Such profiles have different shape at different poloidal locations since diverse are the source and sink terms at play. As sketched in figure 1, the SOL is conventionally divided in (1) the main SOL, above the $\mathrm{X}$-point and surrounding the main plasma, and (2) the divertor $\mathrm{SOL}$, below the X-point and further separated in private (PFR) and common (CFR) flux region. In the main SOL the plasma core represents the source of particles and heat: here the SOL width is a balance between $\|$ transport towards the divertor and $\perp$ transport towards the vessel wall, both acting as sinks. This yields, for a generic SOL quantity $y$, exponentially decaying radial profiles of the form:

$$
y(r)=y_{0} \exp \left(-\frac{r}{\lambda_{y} f_{\mathrm{x}}}\right),
$$

where $y_{0}$ is the separatrix value, $r=\left(R-R_{\text {sep }}\right)_{\text {omp }} \cdot f_{\mathrm{x}} \geqslant 0$ is the radial distance from the separatrix, $f_{\mathrm{x}}$ is the local magnetic flux expansion [8] and the decay length $\lambda_{y}$ approximates the SOL width. In the divertor SOL, instead, the plasma from the main SOL enters the CFR and is lost perpendicularly on either side, towards the vessel wall and in the PFR. This corresponds to a diffusion of the main SOL profiles in the $\perp$ direction, with two consequences: (i) profiles get broader, increasing the wetted width at the targets, and (ii) the peak gets lower and shifts away from the magnetic strike point in the CFR as a result of the roll-over in the PFR [9]. Under these hypotheses, divertor profiles can be described by the convolution of a 
decaying exponential with a Gaussian [10]:

$$
y(r)=\frac{y_{0}}{2} \exp \left(\left(\frac{S_{y}}{2 \lambda_{y}}\right)^{2}-\frac{r}{\lambda_{y} f_{\mathrm{x}}}\right) \operatorname{erfc}\left(\frac{S_{y}}{2 \lambda_{y}}-\frac{r}{S_{y} f_{\mathrm{x}}}\right)+y_{\mathrm{bg}},
$$

where $S_{y}$ is the width of the Gaussian called spreading factor and $y_{\mathrm{bg}}$ is the background value. Negative values of $r$ refer to the PFR. Assuming that trivial details of the magnetic geometry (flaring of flux surfaces and their tilting with respect to divertor targets) can be removed through $f_{\mathrm{x}}$, by definition $\lambda_{y}$ and $S_{y}$ represent the scale lengths of main SOL and divertor transport respectively. Concerning the heat flux, for example, both $\lambda_{q}$ and $S_{q}$, as well as of course $f_{\mathrm{x}}$, will concur setting the overall $\lambda_{\text {wet }}$ at the target. A good estimate of this quantity can be given through the so-called integral width [8]:

$$
\lambda_{\mathrm{wet}}=f_{\mathrm{x}} \lambda_{\mathrm{int}}=f_{\mathrm{x}} \frac{\int\left(q(r)-q_{\mathrm{bg}}\right) \mathrm{d} r}{q_{\max }-q_{\mathrm{bg}}}
$$

which directly links the profile width with $q_{\max }$ that has to respect the material constraints discussed in section 1.1. In particular, for profiles that can be described with equation (2), it is possible to approximate $\lambda_{\text {int }} \simeq \lambda_{q}+1.64 S_{q}$ [11].

\subsection{Scaling laws and extrapolation to ITER}

In order to understand the physics governing the heat flux width and to make predictions for future devices like ITER, one can look for dependencies of $\lambda_{q}$ and $S_{q}$ on plasma control parameters (toroidal magnetic field $B_{\mathrm{T}}$, poloidal magnetic field $B_{\mathrm{P}}$, plasma current $I_{\mathrm{P}}$, edge safety factor $q_{95}$, power entering the SOL $P_{\mathrm{SOL}}$, major radius $R_{0}$, etc.) in existing devices to create scaling laws. With this purpose, a multi-machine database was built using target heat flux profiles measured with infrared (IR) thermography during attached $\mathrm{H}$-mode discharges in tokamaks with D-shaped section (JET, AUG, DIII-D and C-mod) as well as spherical ones (NSTX and MAST). Regression of control parameters identified that the most significant dependency is the one on $B_{\mathrm{P}}$ at the outer midplane: $\lambda_{q}=0.64 B_{\mathrm{P}, \text { omp }}^{-1.15}$ [12]. This scaling law predicts a very small value of $\lambda_{q}=1 \mathrm{~mm}$ for the foreseen ITER $I_{\mathrm{P}}=15 \mathrm{MA}$ scenario. Heat load studies of attached L-mode discharges in JET and AUG [13] showed that $\lambda_{q}^{\mathrm{L} \text {-mode }} \simeq 2 \lambda_{q}^{\mathrm{H} \text {-mode }}$ and has a similar dependence on control parameters. The existence of such a common trend among tokamaks with different vessel shape and aspect ratio would suggest that, if $\lambda_{q}$ depends on any machine-specific feature like, for instance, the magnetic geometry of the divertor, this dependence is weak compared to the one on $B_{\mathrm{P}, \text { omp }}$. This would be coherent with its definition of main SOL quantity given in section 1.3. The trend of $\lambda_{q}$ with $B_{\mathrm{P} \text {,omp }}$ (an therefore with $I_{\mathrm{P}}$, if minor radius $a$ and elongation $k$ are constant) has been reproduced qualitatively by a turbulent model based on blob measurements in L-mode limited discharges in the Tore Supra tokamak [14], as well as in numerical simulation of the edge and SOL turbulence in MAST performed with the ESEL code [15]. Multi-machine scaling laws for limited discharges can be found in [16]. Even though the value of $\lambda_{q}$ in limited discharges can differ from diverted ones, these results confirm the generality of such feature and underline the importance of low field side turbulence localized around the outer midplane [17] in setting $\lambda_{q}$. On the other hand, a cross-machine trend of $S_{q}$ is yet to be found. A scaling law for $S_{q}$ was found for attached L-mode discharges in AUG, highlighting a strong dependence on divertor conditions as electron temperature $T_{\mathrm{e}}$ and density $n_{\mathrm{e}}$ [18]. According to recent experiment on TCV, $S_{q}$ is also insensitive to $f_{\mathrm{x}}$ [19]. Both findings suggest that $S_{q}$, as discussed in section 1.3 , is a divertor quantity and therefore work needs to be done to find common trends among different machines.

With the aim of testing the hypothesis that main SOL and divertor transport can be described with two separated scale lengths, a dedicated experiment, detailed in section 2, was carried out on the TCV tokamak: $\lambda_{q}$ and $S_{q}$ were estimated while scanning the length of the outer divertor leg in L-mode, lower single null (LSN), Ohmic discharges. Experimental findings are compared with different philosophies of transport code simulations (MONALISA [20], SolEdge2D-EIRENE [21], TOKAM $3 X[22,23]$ ) in section 3 to discriminate, among possible mechanisms, the physics responsible for the experimental trends. Section 4 is dedicated to interpretation while section 5 summarizes the conclusions.

\section{Experiment}

\subsection{Motivation}

To understand whether $\lambda_{q}$ is truly insensitive to the magnetic geometry and try to find a scaling parameter for $S_{q}$ are two separate goals that might be achieved within a single experiment. If it was possible to significantly change the size of the divertor while keeping all core plasma parameters constant, according to the aforementioned interpretation, one would expect not to see a variation in $\lambda_{q}$ while $S_{q}$ could effectively increase due to the bigger divertor volume. Most of the tokamaks have limited flexibility in changing the magnetic equilibrium. An exception is represented by the Tokamak à Configuration Variable (TCV). With its elongated vacuum vessel surrounded by 16 independent poloidal field coils, TCV has very good shaping capabilities [6] making it a valuable candidate for this study. The device also benefits from a wide set of edge diagnostics (figure 2(a)): main plasma conditions $\left(n_{\mathrm{e}}^{\mathrm{u}}\right.$ and $\left.T_{\mathrm{e}}^{\mathrm{u}}\right)$ in the core, edge and SOL are monitored with an high resolution Thomson scattering (HRTS) [24] and a reciprocating Langmuir probe (RCP) plunging at the outer midplane [25]. Plasma conditions at the divertor targets $\left(n_{\mathrm{e}}^{\mathrm{t}}\right.$ and $\left.T_{\mathrm{e}}^{\mathrm{t}}\right)$ are assessed with wall-embedded Langmuir probes (LP) [26], while profiles of the heat flux on the target $q_{\text {surf }}^{\mathrm{t}}$ are obtained via the IR thermography system [19].

\subsection{Strategy}

A shot-to-shot scan of the vertical position of the magnetic axis $\left(Z_{\text {mag }}\right)$ was performed in LSN, Ohmic, L-mode, low density $\left(f_{\mathrm{GW}}=n / n_{\mathrm{GW}} \simeq 25 \%-30 \%\right.$, where $n_{\mathrm{GW}}=I_{\mathrm{P}} / \pi a^{2}$ is the Greenwald density limit [27]), attached plasma discharges with 



Figure 2. (a) Cross section of the TCV vacuum vessel with main diagnostics. Magnetic separatrix for short, medium and long outer divertor leg configurations in black, blue and red. (b) Profiles of the SOL parallel connection length from outer midplane to outer divertor target. (c) Parallel connection length (stars), poloidal connection length (triangles) and vertical X-point to target distance (hexagons) as a function of vertical position of magnetic axis.

fixed main plasma shape $\left(R_{0}=89 \mathrm{~cm}, a=22 \mathrm{~cm}, k=1.4\right)$. Ohmic H-mode discharges are not considered in this work. Plasmas at vertical position $Z_{\text {mag }}=-14,0$ and $28 \mathrm{~cm}$, pictured in figure 2(a), were achieved at constant $B_{0}=1.4 \mathrm{~T}$, constant $I_{\mathrm{P}} \simeq 210 \mathrm{kA}$ and therefore constant $B_{\mathrm{P}, \text { omp }} \simeq 0.18 \mathrm{~T}$. Changing $Z_{\text {mag }}$ leads to a variation of $L_{\|}$from the outer midplane to the outer target, whose radial SOL profiles are shown in figure 2(b). The values of $L_{\|}(17 \mathrm{~m}, 19.6 \mathrm{~m}$ and $26.2 \mathrm{~m}$ respectively, averaged over a $5 \mathrm{~mm}$ distance from the magnetic separatrix), are displayed in figure 2(c), together with those of poloidal connection length $L_{\text {pol }}(0.77 \mathrm{~m}, 0.93 \mathrm{~m}$ and $1.21 \mathrm{~m}$ respectively). These three vertical plasma positions correspond to a divertor leg length $L_{\mathrm{div}}$ (vertical X-point to outer target distance) of 21,36 and $64 \mathrm{~cm}$. Each of these quantities increases linearly with $Z_{\mathrm{mag}}$ and therefore they give an equivalent description. In the following $L_{\mathrm{div}}$ will be used and we will refer to these configurations also as short, medium and long leg, respectively. The main deliverable of the experiment is the assessment of the effect of $L_{\mathrm{div}}$ on main plasma and target profiles of $n_{\mathrm{e}}, T_{\mathrm{e}}$ and electron heat flux $q_{\mathrm{e}}$ from LP and $\mathrm{RCP}$, as well as on target $q$ profiles from IR. All discharges used in this dataset are characterized by a variation of core line averaged density $n_{\mathrm{e} \text {,av }}^{\text {core }}$ and of $q_{95}$ within $10 \%$. Every discharge consists of a $500 \mathrm{~ms}$ steady phase in which the magnetic equilibrium is kept as still as possible to help IR measurements, followed by a sweeping phase of few hundreds of $\mathrm{ms}$ in which the divertor leg is moved across different LP to improve the spatial coverage of time-integrated $n_{\mathrm{e}}, T_{\mathrm{e}}$ and $q_{\mathrm{e}}$ target profiles.

\subsection{Upstream plasma conditions}

Measurements of the core and edge plasma properties with HRTS show that, when changing $L_{\text {div }}$ and therefore $L_{\|}$, there is no significant impact on $n_{\mathrm{e}}$ and $T_{\mathrm{e}}$ (figure 3). This result suggests that the goal of matching main plasma conditions, while changing the divertor geometry, was achieved. Due to the sensitivity of Thomson scattering channels in the edge region, experimental data in the main SOL are available only for plasmas with medium divertor leg. When comparing target to main SOL profiles for the different values of $L_{\text {div }}$ we take as working assumption that, given the good match in the core and edge, $n_{\mathrm{e}}$ and $T_{\mathrm{e}}$ match also in the main SOL. In figures 4(a) and (b) respectively, outer midplane measurements of $n_{\mathrm{e}}$ and $T_{\mathrm{e}}$ performed with a reciprocating double probe system [25] (RCP, green diamonds) are superimposed to target profiles from wall LP (blue dots) for the medium leg configuration. Outer midplane and target density profiles are in good agreement, suggesting no strong variation of $n_{\mathrm{e}}$ in terms of peak value nor of decay length along $L_{\|}$. On the other hand $T_{\mathrm{e}}$ drops on average by a factor of $\simeq 2$, 



Figure 3. Thomson scattering, main plasma, profiles of (a) $n_{\mathrm{e}}$ and (b) $T_{\mathrm{e}}$ as a function of the radial distance from the outer midplane separatrix for $L_{\mathrm{div}}=21 \mathrm{~cm}, 36 \mathrm{~cm}$ and $64 \mathrm{~cm}$ (black, blue and red triangles respectively).


Figure 4. Outer midplane, reciprocating Langmuir probe (green diamonds) and outer target Langmuir probes (blue circles) profiles of (a) $n_{\mathrm{e}}$ and (b) $T_{\mathrm{e}}$ as a function of the radial distance from the outer midplane separatrix for the medium divertor leg configuration.

indicating that the static pressure $P_{\mathrm{e}}=n_{\mathrm{e}} T_{\mathrm{e}}$ is not conserved along the outer SOL. If one instead considers the total pressure $P_{\mathrm{e}}^{*}=n_{\mathrm{e}} T_{\mathrm{e}}\left(1+M^{2}\right)$ and assumes $M=1\left(v_{\|}=c_{\mathrm{s}}\right)$ near the target, according to the Bohm criterion, conservation is fulfilled: $P_{\mathrm{e}}^{* \mathrm{t}}=2 n_{\mathrm{e}}^{\mathrm{t}} T_{\mathrm{e}}^{\mathrm{t}} \simeq P_{\mathrm{e}}^{*, \mathrm{u}}=n_{\mathrm{e}}^{\mathrm{u}} T_{\mathrm{e}}^{\mathrm{u}}$. This result suggests that, at the outer target, the plasma is in attached condition.

\subsection{Target plasma conditions}

The wall LP system for the outer divertor target consists in a single array of 26 cylindrical domed probes protruding by $1 \mathrm{~mm}$, with a diameter of $4 \mathrm{~mm}$ and a spatial resolution of $11 \mathrm{~mm}$ [26]. The acquisition is sampled at $500 \mathrm{kHz}$ and $I-V$ characteristics are averaged over $50 \mathrm{~ms}$, shorter than the frequency at which the magnetic strike point is swept across two neighbouring probes. A four parameters fit is performed on the $I-V$ characteristics, to account for the sheath expansion in the saturation current branch. LP measurements show an effect of the divertor magnetic geometry on $n_{\mathrm{e}}, T_{\mathrm{e}}$ and $q_{\mathrm{e}}$ : width, shape and amplitude of the outer target profiles change with the length of the outer divertor leg. For the sake of readability, all shown profiles are obtained binding data from three to four discharges repeated for the same value of $L_{\mathrm{div}}$ and in similar conditions: markers represent the average value of each dataset, error bars the corresponding standard deviation. Profiles of $n_{\mathrm{e}}, T_{\mathrm{e}}$ and $q_{\mathrm{e}}$ are plotted as a function of the radial distance from the magnetic separatrix $\left(R-R_{\text {sep }}\right)$ at the outer midplane: it should be remembered that LP profiles are measured at the divertor target and then remapped at the outer midplane along magnetic flux $(\psi)$ surfaces in order to allow the comparison of magnetic equilibria with different $f_{\mathrm{x}}$. A least square fit to equation (2), represented by the solid lines, is performed to estimate the transport scale lengths $\left(\lambda_{y}, S_{y}\right)$ : here markers represent the value obtained by fitting the whole dataset for a given condition, without binding the data, while error bars correspond to the accuracy of the fit.

Figure 5(a) shows $n_{\mathrm{e}}$ profiles normalized to their maximum value to help visualizing shape variations. The information about 



Figure 5. (a) Normalized outer target Langmuir probes profiles of $n_{\mathrm{e}}$ as a function of radial distance from the outer midplane separatrix for short, medium and long outer divertor leg configurations in black, blue and red. Density (b) peak value, (c) decay length and (d) spreading factor as a function of outer divertor leg length. Green diamonds for outer midplane reciprocating Langmuir probe data.

the corresponding peak value is given in figure $5(\mathrm{~b}): n_{\mathrm{e}, \max }=$ $5.2 \times 10^{18} \mathrm{~m}^{-3}, 6.7 \times 10^{18} \mathrm{~m}^{-3}$ and $6.1 \times 10^{18} \mathrm{~m}^{-3}$ correspond to $18 \%, 23 \%$ and $21 \%$ of $n_{\mathrm{e}, \text { av }}^{\text {core }}$ respectively, indicating similar core-to-target drop regardless of the divertor leg length. For the medium leg configuration, the value of $n_{\mathrm{e}, \max }$ is consistent with the one at the separatrix, $n_{\mathrm{e}}^{\text {sep }}$, measured by the RCP at the outer midplane (green diamond) meaning that no density drop occurs along the outer SOL. These results suggest that there is not a strong effect of $L_{\text {div }}$ on the density peak value. If one instead considers width and shape of the profiles, the impact of changing the divertor geometry is stronger: a monotonic trend of the density decay length $\lambda_{n_{\mathrm{e}}}$ with $L_{\text {div }}$ (figure 5(c)) is detected, with a factor of $\simeq 3$ increase over the explored range. The value of $\lambda_{n_{\mathrm{e}}}$ for the medium leg configuration is $\simeq 1.4$ times bigger than the one measured by the RCP at the outer midplane (green diamond), suggesting that a broadening of the density profile occurs on the path from the outer midplane to the target. The density spreading factor $S_{n_{\mathrm{e}}}$ (figure 5(d)) shows no trend with $L_{\mathrm{div}}$, with values in the range from $\simeq 2.5$ to $\simeq 4.5 \mathrm{~mm}$. Interestingly the main SOL transport scale length $\lambda_{n_{\mathrm{e}}}$ has a stronger relative variation than the divertor one $S_{n_{\mathrm{e}}}$, when increasing the outer divertor leg length. It should be noted that $n_{\mathrm{e}}$ profiles for the short and medium leg configurations exhibit an asymmetric background, which is non null in the far CFR. These shoulders, unlike the density-dependent ones observed for instance in $[28,29]$, correspond to positions on the outer divertor target that are not seamlessly connected to the inner divertor target since magnetic field lines intercept the vacuum vessel either at the top of the machine or at the outer wall. For this reason, such locations are not taken into account in the fitting procedure, which was performed up to $R-R_{\text {sep }}=10 \mathrm{~mm}$ and $15 \mathrm{~mm}$ for short and medium leg, respectively.

Figure 6(a) shows the corresponding normalized target $T_{\mathrm{e}}$ profiles. Unlike density, $T_{\mathrm{e}}$ exhibits a clear drop in peak value and a less marked broadening of profiles (at least in the CFR) with increasing $L_{\mathrm{div}}$. In fact, the peak value $T_{\mathrm{e}, \max }$ (figure 6(b)) is reduced by a factor of $\simeq 2$ with $L_{\text {div }}$, from $\simeq 20.3$ to $\simeq 11.2 \mathrm{eV}$. The value for the medium leg configuration ( $\simeq 17.8 \mathrm{eV}$ ) corresponds to $40 \%$ of $T_{\mathrm{e}}^{\mathrm{sep}}$ measured by the RCP at the outer midplane (green diamond) indicating a $T_{\mathrm{e}}$ drop along the SOL. The target temperature decay length $\lambda_{T_{\mathrm{e}}}$, whose values are in the range $20-35 \mathrm{~mm}$, is not straightforwardly affected by $L_{\text {div }}$, as no trend stands out of the error bars 

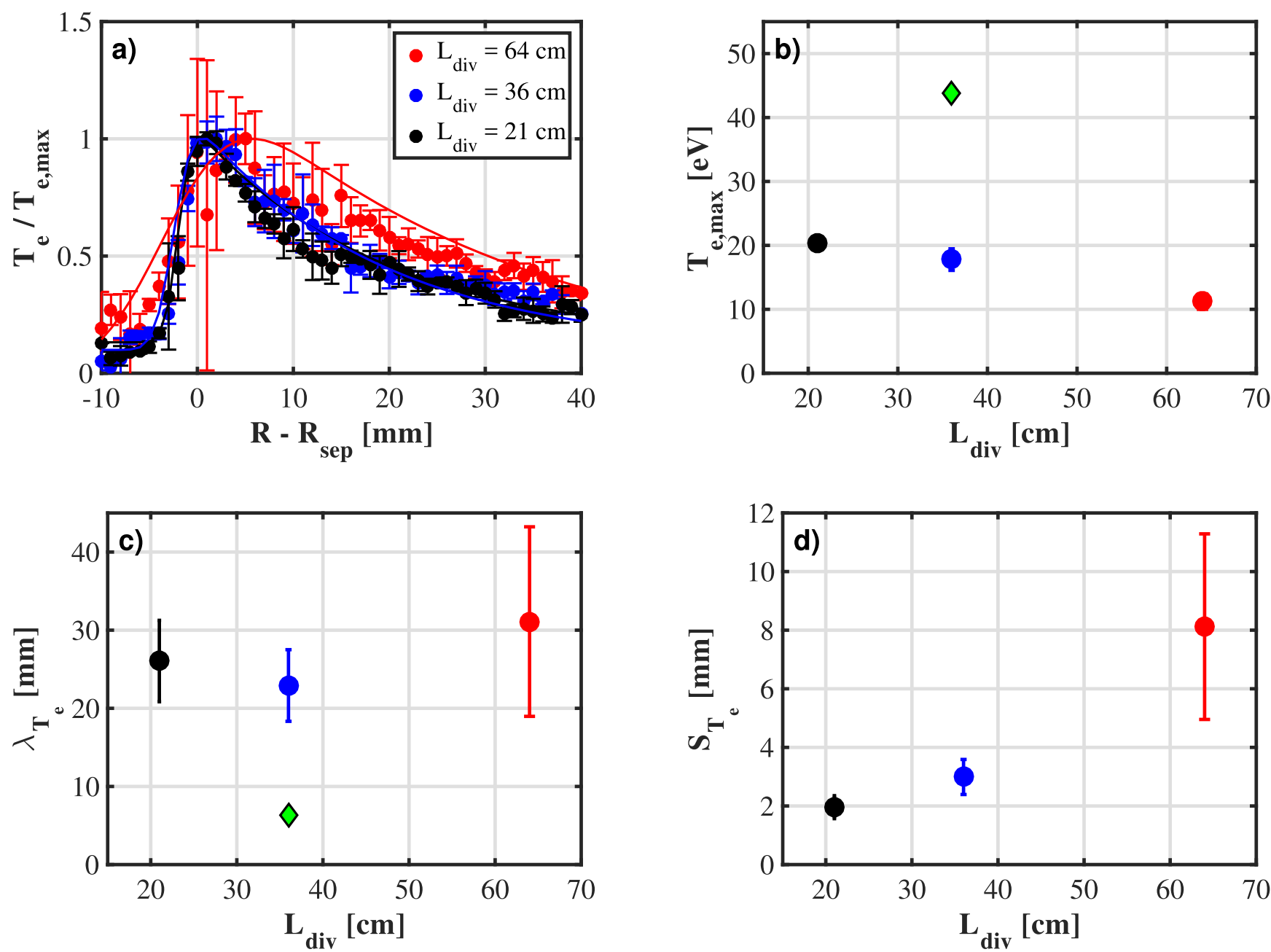

Figure 6. (a) Normalized outer target Langmuir probes profiles of $T_{\mathrm{e}}$ as a function of radial distance from the outer midplane separatrix for short, medium and long outer divertor leg configurations in black, blue and red. Temperature (b) peak value, (c) decay length and (d) spreading factor as a function of outer divertor leg length. Green diamonds for outer midplane reciprocating Langmuir probe data.

(figure $6(\mathrm{c})$ ). The value for the middle configuration is $\simeq 3$ times bigger than the one measured by the RCP (green diamond), suggesting a strong broadening of the $T_{\mathrm{e}}$ profile along the outer SOL. Unlike $\lambda_{T_{\mathrm{e}}}$, the temperature spreading factor $S_{T_{\mathrm{e}}}$ changes monotonically with $L_{\text {div }}$ (figure 6(d)), with a factor of $\simeq 2$ to $\simeq 5$ increase between short and long leg configurations, within the big error bar of the latter. This would mean that, for temperature, a very long divertor leg has a stronger effect on the divertor transport scale length than on the main SOL one. Finally the corresponding $q_{\mathrm{e}}$ profiles are shown in figure 7(a), where a clear broadening with $L_{\mathrm{div}}$ can be detected. With $q_{\mathrm{e} \text {, max }}$ values of $184.8 \mathrm{kWm}^{-2}, 260.4 \mathrm{~kW} \mathrm{~m}^{-2}$ and $87.4 \mathrm{~kW} \mathrm{~m}^{-2}$ respectively, a factor of $\simeq 2$ drop between short and long leg configurations is measured (figure 7(b)). These data correspond to $\simeq 60 \%$ of the heat flux measured by the IR thermography (empty squares) [19], which is the total heat flux absorbed by the target and therefore comprehends not only the electronic component but also the ionic one as well as radiation. According to standard sheath theory, the heat flux can be estimated from LP data as $q=\gamma k T_{\mathrm{e}} \Gamma_{\mathrm{e}}=\gamma k T_{\mathrm{e}} n_{\mathrm{e}} c_{\mathrm{s}}$, where $\gamma$ is the sheath heat transmission coefficient. If equally important contribution from electrons and ions is assumed $\left(T_{\mathrm{i}}=T_{\mathrm{e}}\right)$, then one should take $\gamma=8$ [7]. Given that the assumption of $T_{\mathrm{i}}=0$ was made ( $\gamma=5$, electron heat flux only), such difference is consistent with the $5 / 8 \simeq 60 \%$ discrepancy between LP and IR. The heat flux decay length $\lambda_{q_{\mathrm{e}}}$ (figure 7(c)) undergoes a factor of $\simeq 2$ increase with $L_{\text {div }}$ which is consistent with $\lambda_{q}^{\mathrm{IR}}$ (empty squares). The value measured for the medium leg configuration is $\simeq 1.4$ times larger than the corresponding $\lambda_{q_{o}}^{\mathrm{RCP}}$ measured at the outer midplane (green diamond) suggesting that, besides flux expansion and divertor spreading, the heat flux profile broadens along the outer SOL. For the heat flux spreading factor $S_{q_{\mathrm{e}}}$ instead, no trend can be detected within the sensibility of the two diagnostics (figure 7(d)). Here values are in the range of 2-3 mm and, especially for the long leg configuration, error bars are comparatively big. To summarize, according to LP measurement, a longer divertor leg causes the broadening of $q_{\mathrm{e}}$ profiles and the corresponding drop of the peak value. Interestingly, as observed for $n_{\mathrm{e}}$ profiles, both diagnostics highlight a stronger variation of the main SOL transport scale length $\lambda_{q}$ compared to the divertor one $S_{q}$ when modifying the divertor geometry. 



Figure 7. (a) Normalized outer target Langmuir probes profiles of $q_{\mathrm{e}}$ as a function of radial distance from the outer midplane separatrix for short, medium and long outer divertor leg configurations in black, blue and red. Heat flux (b) peak value, (c) decay length and (d) spreading factor as a function of outer divertor leg length. Empty squares for target infrared data. Green diamond for outer midplane reciprocating Langmuir probe data.

\section{Modelling of SOL transport}

This section concerns the modelling of the aforementioned divertor leg experiment. Different codes including different physics, based on different assumptions have been employed. The results point out that the key to capture the different features of the effect of divertor geometry on SOL quantities, with focus on heat flux profiles, is the assumed degree of (dis)homogeneity of transport. The first attempt consists in using diffusive models with uniform transport coefficients in the whole simulation domain. Then a radial dependence $D_{\perp}(r), \chi_{\perp}(r)$, arbitrary or inferred from experimental measurements, can be assumed. A step further consists in modelling the so-called ballooned transport in which perpendicular transport is enhanced in the surroundings of the outer midplane: this can be done with transport coefficients that change also in the poloidal direction: $D_{\perp}(r, \theta), \chi_{\perp}(r, \theta)$. Last and more complex approach is to use a first principle turbulent model in which particle fluxes are calculated self-consistently and therefore the corresponding effective perpendicular transport coefficient would differ from point to point in the simulation domain: $D_{\perp, \text { eff }}(R, Z)$.

\subsection{Homogeneous perpendicular transport}

A simple approach to the modelling of SOL transport is to assume that the plasma travels mainly in the parallel direction while undergoing diffusion in the perpendicular one. These are the basic assumptions of MONALISA [20], a Monte Carlo code in which energy packets with parallel velocity $v_{\|}=c_{\mathrm{S}}$ from a Maxwellian distribution of width $T$ freely stream along $\vec{B}$ while diffusing perpendicularly under an arbitrary coefficient $D_{\perp}$ which is uniform in the entire domain of the simulation. Although the physics of transport is as simple as it can possibly get, featuring the real magnetic geometry from experimental $\psi$ maps and the actual tokamak wall, MONALISA can well reproduce experimental $q$ profiles, as demonstrated by the black solid line in figure 8 , where numerical and experimental profiles for the medium leg configuration are superimposed. Attempts to model the divertor leg experiment with such code give mixed results: simulations with different $L_{\text {div }}$ at constant $D_{\perp}=0.5 \mathrm{~m}^{2} \mathrm{~s}^{-1}, T=40 \mathrm{eV}, I_{\mathrm{P}}=210 \mathrm{kA}$ and $B_{\mathrm{T}}=1.4 \mathrm{~T}$, show no increase in $\lambda_{q}$ (figure 9(a), solid black line) while a monotonically increasing $S_{q}$ is predicted (figure 9(b)). Interestingly, MONALISA results fit well to IR experimental data 


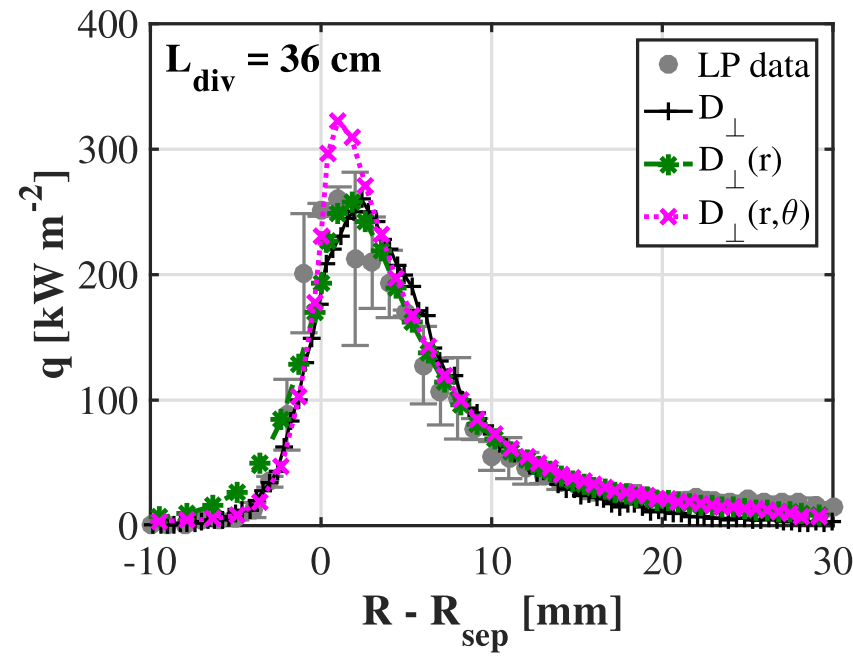

Figure 8. Numerical heat flux profile at the outer divertor target for the medium leg configuration $\left(L_{\mathrm{div}}=36 \mathrm{~cm}\right)$ : constant $D_{\perp}$ (solid black line), $D_{\perp}(r)$ and $\chi_{\perp}(r)$ (dashed green line), $D_{\perp}(r, \theta)$ and $\chi_{\perp}(r, \theta)$ (dotted magenta line). Grey markers refer to experimental LP data shown in figure 7(a).

(squares) for $L_{\mathrm{div}}=21$ and $36 \mathrm{~cm}$, but the factor of 2 increase in $\lambda_{q}$ for $L_{\mathrm{div}}=64 \mathrm{~cm}$, as well as the non monotonic trend for $S_{q}$ cannot be captured. This leads, for the latter configuration, to a strong overestimation of the ratio $S_{q} / \lambda_{q}$ (figure 9(c)) which is an indicator of the degree of asymmetry: when $S_{q} / \lambda_{q}$ approaches 1, profiles become Gaussian-like while in experiments $\left(S_{q} / \lambda_{q}=0.3\right)$ they are much steeper on the PFR side than the CFR one. Simulations performed with the more sophisticated SolEdge2D-EIRE code package, described in section 3.2, assuming constant transport coefficients, yield trends in qualitative agreement with MONALISA: $\lambda_{q}$ is constant with $L_{\mathrm{div}}$ while $S_{q}$ increases monotonically. Even though, in this kind of simulations, the values of the two scale lengths do not match the experiment for the long leg case, and therefore the information about the relative importance of radial transport in the main SOL and divertor SOL cannot be extracted, it is worth noting that $\lambda_{\text {int }}$ is in good agreement (figure 9(d)). This result suggests that, even for modified divertor geometries, this model can still give a good indication about the average width of heat flux profiles.

\subsection{Radially dependent perpendicular transport}

A more sophisticated approach consists in assuming that perpendicular transport coefficients, and therefore the corresponding fluxes, can be inhomogeneous in the radial direction: e.g. for density $\Gamma_{n, \perp}=-D_{\perp}(r) \nabla_{\perp} n$. If the variation of $D_{\perp}(r)$ and $\chi_{\perp}(r)$ is not strong, main SOL profiles can still be fit with a decaying exponential. Simulations under this assumption were performed with a more powerful modelling tool: the SolEdge2D-EIRENE code package [21]. The treatment of the plasma is undertaken by the SolEdge2D module which solves the fluid equations (mass, momentum and energy conservation) for two species, electrons and singly charged ions, assuming quasi-neutrality $\left(n_{\mathrm{e}}=n_{\mathrm{i}}\right)$ and ambipolarity $\left(u_{\mathrm{e}}=u_{i}\right)$. A peculiarity of SolEdge2D is that the simulation domain extends up to the first wall where the interaction of the plasma with material surfaces is also modelled. The EIRENE Monte Carlo code, on the other hand, treats neutrals through atomic and molecular reactions as well as recycling [21]. For this study, a fit to experimental outer midplane profiles of $n_{\mathrm{e}}$ and $T_{\mathrm{e}}$ from RCP and HRTS, available in the SOL region only for the configuration with $L_{\mathrm{div}}=36 \mathrm{~cm}$ (medium leg), is used as input for all three configurations. This was done under the ansatz that outer midplane profiles would overlap in the SOL region regardless the value of $L_{\mathrm{div}}$ as they do for the edge and core region for which there are HRTS measurements, as discussed in section 2.3. Radial profiles of the perpendicular transport coefficients $D_{\perp}(r)$ and $\chi_{\perp}(r)$ are therefore produced by the code as $n_{\mathrm{e}}$ and $T_{\mathrm{e}}$ are calculated everywhere in the domain while keeping the imposed outer midplane profiles. As shown by the green dashed line in figure 8 , the simulated heat flux profile for the medium leg configuration matches well the one obtained with MONALISA (black solid line) as well as the experimental LP data. The results in terms of profile widths, depicted by the green dashed lines in figure 9, are overall similar to those obtained with homogeneous perpendicular transport coefficients. SolEdge2D-EIRENE, like MONALISA, yields an almost constant $\lambda_{q}$ and an increasing $S_{q}$. For $L_{\mathrm{div}}=21 \mathrm{~cm}$ and $36 \mathrm{~cm}, S_{q}$ basically coincides while for $L_{\text {div }}=64 \mathrm{~cm}$ SolEdge2D-EIRENE predicts a $30 \%$ higher value. Such an increase is most probably an effect of the temperature distribution along the divertor leg: a bigger drop and therefore a smaller $v_{\|}$in the vicinity of the target allows a longer time for perpendicular diffusion. This translates in a ratio $S_{q} / \lambda_{q}=1.13$ which is even further from the experimental trend. The reason of such a big divertor spreading can be visualized in figure 10(a) where a 2D map of the perpendicular particle flux $\Gamma_{n, \perp}$ for the long legged plasma is shown. Here inward transport in the PFR is non negligible along the entire divertor length and in the vicinity of the target is as strong as the outward one in the CFR resulting in Gaussian-like profiles. Similarly to section 3.1, even though the $\lambda_{q}$ trend is underestimated and the one of $S_{q}$ overestimated, $\lambda_{\text {int }}$ found in simulations is in good agreement with the data.

\subsection{Ballooned perpendicular transport}

One might instead suppose that perpendicular transport coefficients can vary both in the radial and poloidal direction: $D_{\perp}(r, \theta), \chi_{\perp}(r, \theta)$. This hypothesis allows to mimic the socalled ballooning, meaning the enhanced turbulent transport localized in the vicinity of the outer midplane observed, for instance, in limited plasmas on Tore Supra [17] and in diverted ones on Alcator C-mod [30]. Radius-dependent transport coefficients discussed in section 3.2 are modified by adding a Gaussian dependence in the poloidal direction which has the effect of reducing perpendicular transport away from the outer midplane. The corresponding target heat flux profile (magenta dotted line in figure 8 ) is characterized by a smaller roll-over in the PFR, and therefore by a higher peak value, when compared to the one obtained with SolEdge2D-EIRENE simulations with 

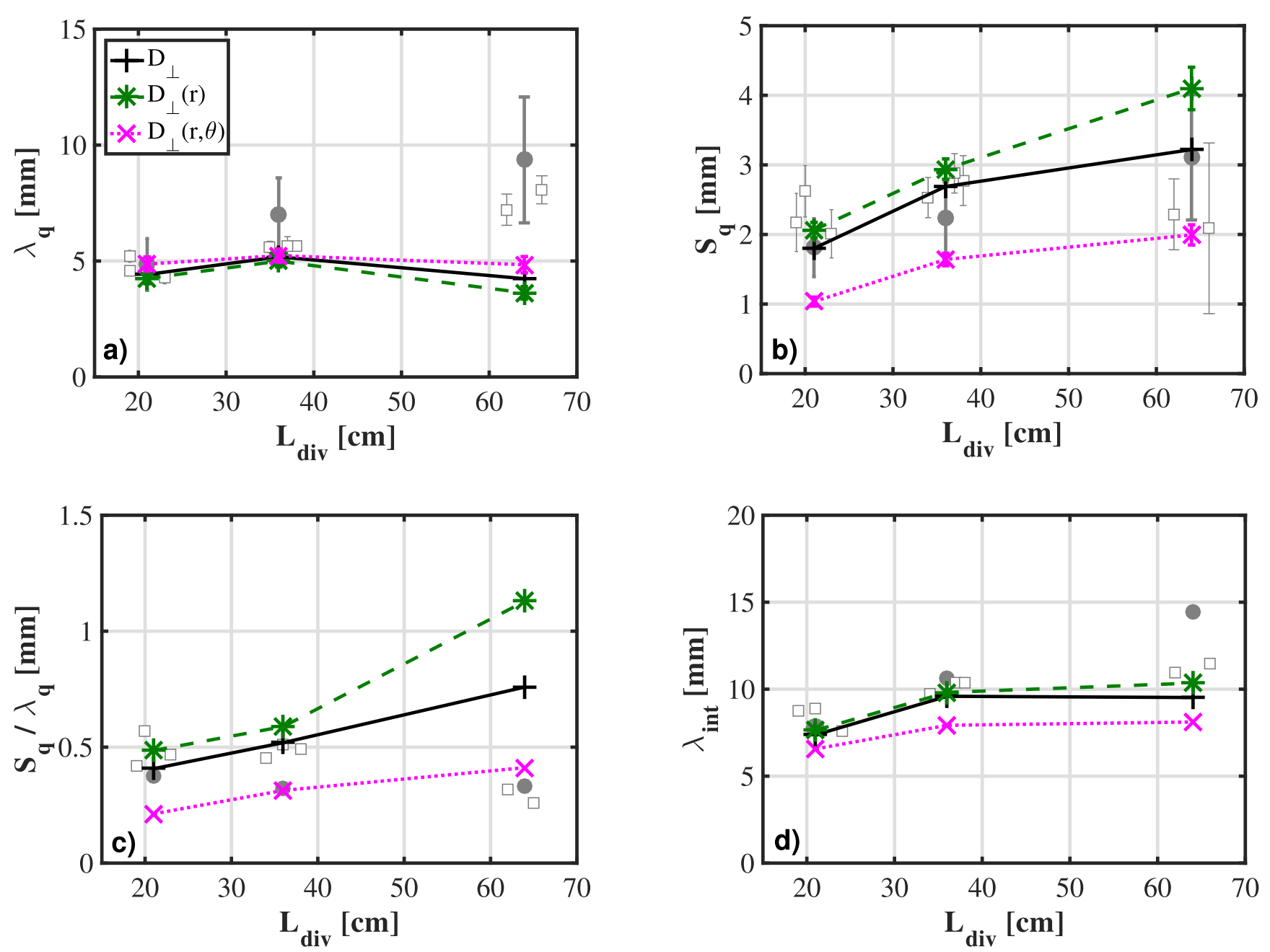

Figure 9. Numerical results of (a) $\lambda_{q}$, (b) $S_{q}$, (c) $S_{q} / \lambda_{q}$ and d) $\lambda_{\text {int }}$ as a function of $L_{\text {div }}$ for constant $D_{\perp}$ (solid black line), $D_{\perp}(r)$ and $\chi_{\perp}(r)$ (dashed green line), $D_{\perp}(r, \theta)$ and $\chi_{\perp}(r, \theta)$ (dotted magenta line). Grey markers refer to experimental data shown in figure 7 .
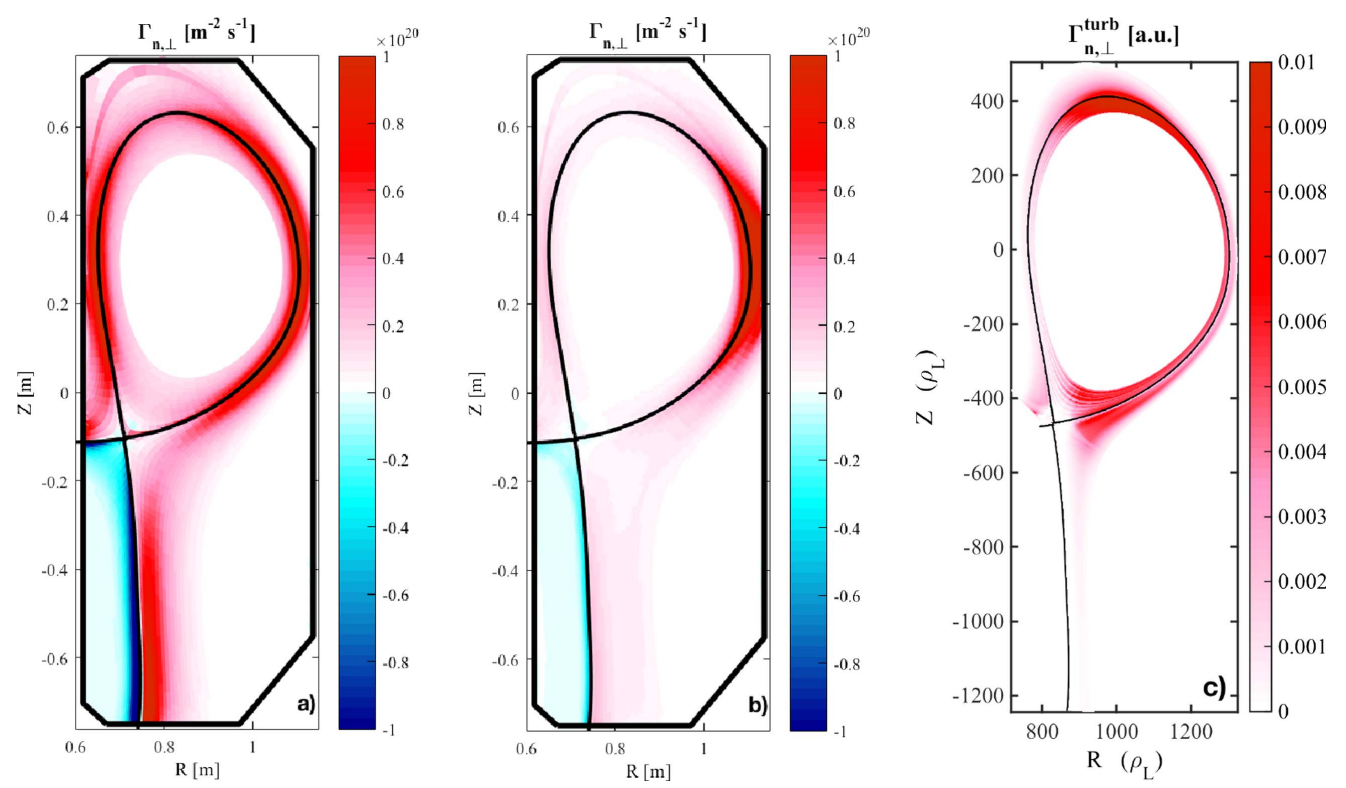

Figure 10. Numerical 2D maps of $\Gamma_{n, \perp}$ for (a) $D_{\perp}(r)$ and (b) $D_{\perp}(r, \theta)$ from SolEdge2D-EIRENE diffusive simulations, and (c) from TOKAM3X turbulent simulations (fluctuating component). 

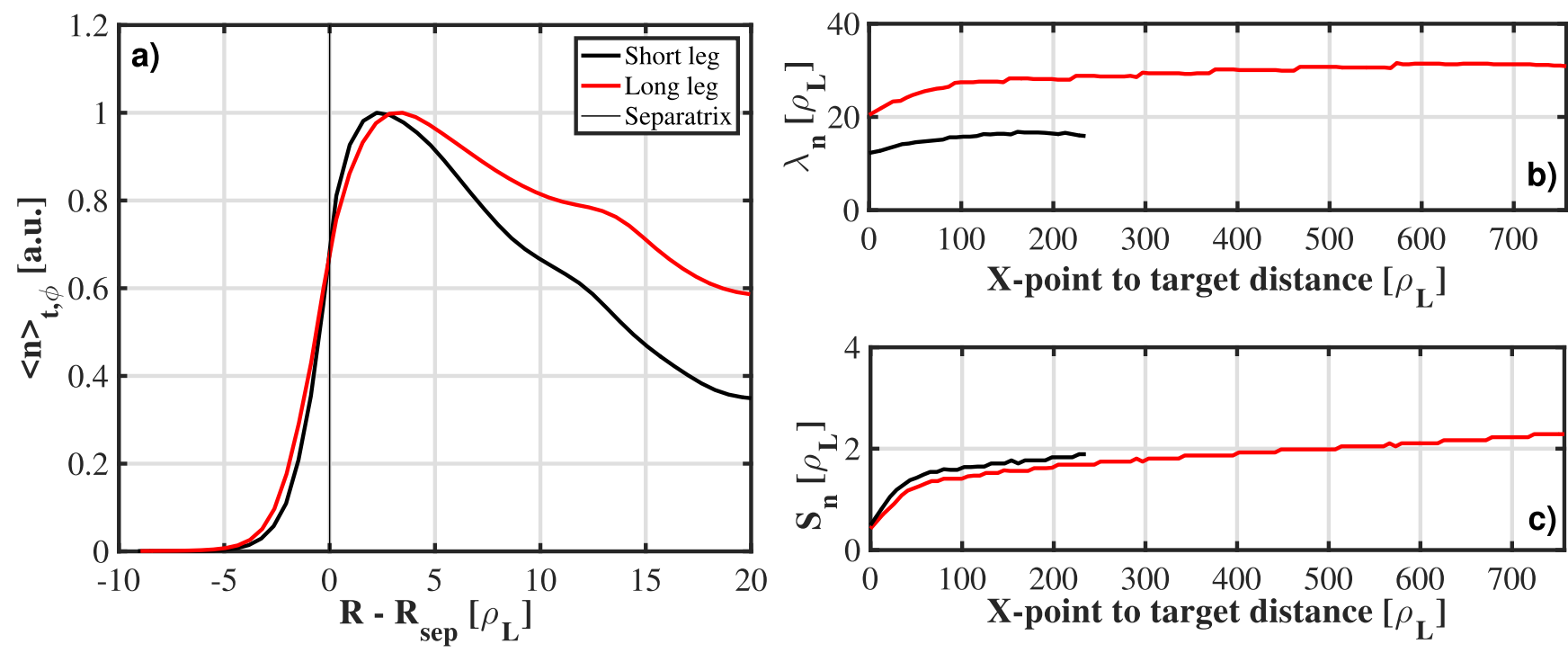

Figure 11. TOKAM $3 \mathrm{X}$ profiles of (a) density averaged over time and toroidal angle as a function of radial distance from the outer midplane separatrix, (b) $\lambda_{n}$ and (c) $S_{n}$ as a function of the poloidal X-point to target distance (black for the short leg plasma, red for the long leg one).

radially dependent perpendicular transport coefficients (green dashed line). The width of the profiles is therefore impacted (figure 9, dotted magenta lines): while $\lambda_{q}$ is found to be basically the same as in previous simulations and therefore not to change with $L_{\mathrm{div}}, S_{q}$ is reduced by a factor of $\simeq 2$ coherently with a smaller perpendicular transport in the divertor. In fact, as shown in figure 10 (b), in these simulations $\Gamma_{n, \perp}$ is strongly localized around the outer midplane, while it is damped everywhere else with respect to section 3.2 and figure 10(a). This translates in a stronger profile asymmetry for the long leg case $\left(S_{q} / \lambda_{q}=0.4\right)$ which gets much closer to what is found in experiments. As a consequence of the reduction of $S_{q}$, also $\lambda_{\text {int }}$ gets smaller. Ballooning clearly represents an improved description of perpendicular transport but it is still however not possible to reproduce the experimental trend in $\lambda_{q}$.

\subsection{Turbulent transport}

An alternative approach to modelling edge and SOL is to abandon the simplified diffusive picture and to take into account the turbulent nature of transport. This can be done with TOKAM3X, a 3D fluid turbulent code solving a set of drift-reduced conservation equations for mass, parallel momentum, electric charge and energy for electrons and ions, in an electrostatic approximation [22, 23]. TOKAM3X gives a multi-scale description of turbulence in plasma edge and SOL. The equations in its model are normalized using the ion Larmor radius $\rho_{\mathrm{L}}$ and the inverse of the ion gyrofrequency $\omega_{\mathrm{c}}$ respectively as characteristic length and time scales. A fluxdriven approach is adopted, in which a particle source is imposed in the closed field line region, and the sink mechanism is provided by the sheath boundary conditions in the SOL. TOKAM3X can handle non-trivial magnetic geometries as the ones investigated in this work, simulating the plasma behaviour on both open and closed flux surfaces in a reduced, TCV-like, geometry. This means that the geometry used in the code is a homothetic transformation (i.e. a homogeneous compression) of the reference TCV equilibria, which helps reducing the computational time. A physical time span corresponding to $\sim 4$ times the confinement time is typically simulated, in order to reach a full development of turbulence. The simulations presented here are obtained with the isothermal version of the code and therefore the discussion is focused on density profiles. Interestingly, the fluctuating component of the perpendicular particle flux $\Gamma_{n, \perp}^{\mathrm{turb}}=\left\langle\tilde{n} \tilde{v}_{E \times B}^{r}\right\rangle_{t, \phi}$ simulated by TOKAM3X (shown in figure 10(c)) suggests the absence of transport in the PFR as well as in the immediate vicinity of the magnetic separatrix in the CFR. This result is in contrast with what is predicted under the diffusive ansatz by SolEdge2D-EIRENE simulations (figures 10(a) and (b)). Turbulent perpendicular transport is instead strong in the main plasma, where it extends from the outer midplane up to the top of the equilibrium as well as close to the X-point, and non negligible along the far CFR. The exhibited strong asymmetry in divertor transport has an effect on the shape of target profiles: figure 11(a) shows density, averaged over time and toroidal angle, $\langle n\rangle_{t, \phi}$ as a function of radial distance from the outer midplane separatrix. As for the experimental data, profiles are renormalized to the maximum value to better display the change in shape. For the short leg case $\left(L_{\mathrm{div}}=21 \mathrm{~cm}-\right.$ like, black line) $\lambda_{n}=15.9 \rho_{\mathrm{L}}$ and $S_{n}=1.9 \rho_{\mathrm{L}}$, while for the long leg case $\left(L_{\text {div }}=64 \mathrm{~cm}\right.$-like, red line) $\lambda_{n}=30.9 \rho_{\mathrm{L}}$ and $S_{n}=2.3 \rho_{\mathrm{L}}$ respectively. These results imply that the profile asymmetry $\left(S_{q} / \lambda_{q}=0.12\right.$ and 0.07 respectively) is stronger than the one found experimentally or in SolEdge2D-EIRENE ballooned simulations. Moreover the factor of 2 increase in $\lambda_{n}$, that was not captured by diffusive models, is in qualitative agreement with the increase of $\lambda_{n_{\mathrm{e}}}$ measured with LP, which is believed to be the driver of the factor of $\simeq 2$ in $\lambda_{q}$ exhibited by both LP and IR data. When comparing the trend in peak value $n_{\text {max }}$ instead, the agreement between TOKAM3X and LP data is quantitative, with a factor of 1.16 drop between short and long leg case. Such difference with respect to non turbulent simulations can be understood by looking at 2D maps of 

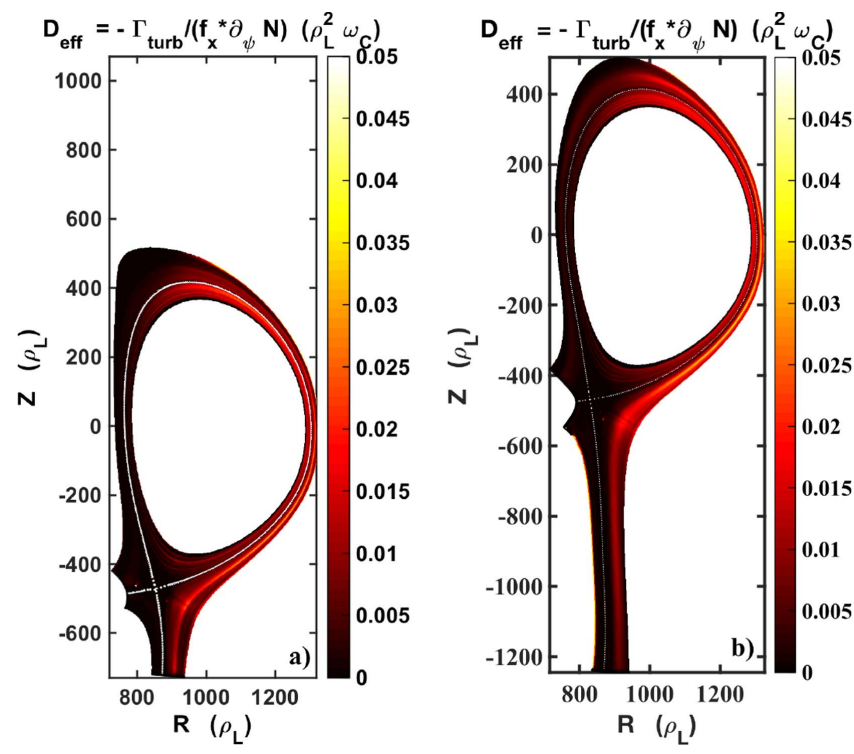

Figure 12. TOKAM $3 X$ 2D maps of the effective perpendicular transport coefficient for (a) the short leg and (b) the long leg simulations.

the effective diffusion coefficient calculated as $D_{\perp, \text { eff }}=$ $-\Gamma_{n, \perp}^{\text {turb }} / \nabla_{\perp} n$ and corrected by the magnetic flux expansion. Figure 12 compares $D_{\perp, \text { eff }}$ for the short and long leg scenarios highlighting some common features. Perpendicular transport is enhanced at the outer midplane and it clearly extends poloidally upwards towards the top of the main plasma and downwards close to the X-point, consistently with the ballooning character of turbulent transport [17,30]. The big difference with respect to the aforementioned ballooned SolEdge2DEIRENE simulations is represented by a non negligible value of $D_{\perp, \text { eff }}$ all along the CFR, far from the magnetic separatrix, causing a non symmetric divertor spreading which translates in a wider $\lambda_{n}$ for a longer leg. Conversely, $D_{\perp, \text { eff }}$ is so small in the PFR that no big difference in $S_{n}$ is observed. Figures 11(b) and (c) show the evolution of $\lambda_{n}$ and $S_{n}$ along the poloidal path from the X-point to the target. On the one hand $\lambda_{n}$ is found to be already different at the divertor entrance (X-point) and to increase gently along the respective leg lengths. On the other $S_{n}$ exhibits the same behaviour in both trend and amplitude, suggesting that most of the broadening takes place in the first $50 \rho_{\mathrm{L}}$ below the X-point and afterwards it gently increases over the allowed divertor leg length, with a difference of less than $20 \%$ at the target. Such an increase of both perpendicular transport scale lengths is related to the presence of a strong steady convective cell around the X-point in TOKAM3X simulations [23]: this causes the mean field transport in this region to be strong, despite the fact that its fluctuating component, as was shown in figure 10(c), is almost zero.

\section{Interpretation}

When attempting to reproduce the divertor leg experiment carried out in TCV with simulations, it is observed that in modified magnetic configurations, as the long leg one presented in this work, a turbulent description is required. Modelling with TOKAM3X shows that turbulent perpendicular transport, believed to be important and setting $\lambda_{q}$ only in the main plasma, is instead strong also all along the divertor leg in the far CFR, while almost no transport is predicted in the vicinity of the separatrix and in the PFR. This mechanism is most probably at play also in the more "traditional' divertor configurations (short and medium leg) although less evident because of the short X-point to target distance. For this reason a diffusive picture efficiently describes these equilibria while strongly underestimates $\lambda_{q}$ for the long leg plasma. For short-legged plasmas though, both a very simplified isothermal model with homogeneous diffusion and a more complex fluid code, with radial-dependent perpendicular transport coefficients inferred from experimental measurements, match reasonably well with target heat flux profiles.

In the presence of asymmetric divertor transport, when profiles are fit to equation (2), which is based on the hypothesis of symmetric diffusion in the divertor region, a bigger $\lambda_{y}$ is found even though the broadening described here is due to transport occurring below the X-point and, in this framework, should therefore be described by $S_{y}$. Therefore, the current parametrisation has to be generalized for the case of asymmetric divertor perpendicular transport: this could be attempted by introducing a third scale length $S_{\mathrm{CFR}}$ describing the observed, non negligible, transport in the CFR as opposed to $S_{\mathrm{PFR}}<S_{\mathrm{CFR}}$ accounting for the weak transport in the PFR. In this framework, $\lambda_{q}$ would represent only the main SOL transport and should not be expected to change with $L_{\mathrm{div}}$, while the broadening of target profiles observed in the experiment, which is a divertor effect, would be captured by $S_{\mathrm{CFR}}$. In any case a clear role of turbulent, divertor localized, asymmetric perpendicular transport in setting the overall SOL width has to be taken into account for such magnetic geometries.

\section{Conclusions}

This work constitutes a study of the effect of changing the length of the outer divertor leg on divertor power exhaust in low density, LSN, Ohmic, attached, L-mode plasmas discharges in TCV. Experimental IR and LP measurements of the outer target heat flux profiles show a monotonic increase of $\lambda_{q}$ with $L_{\mathrm{div}}$ with a factor of $\simeq 2$ difference over the explored range, at constant $B_{\mathrm{P}, \text { omp }}$. LP analysis of target profiles suggests that such broadening is driven by a strong increment in $\lambda_{n_{\mathrm{e}}}$ while $\lambda_{T_{\mathrm{e}}}$ is only slightly increasing. On the other hand, no trend of $S_{q}$ with $L_{\text {div }}$ is detected within the resolution of the two diagnostics. Modelling efforts showed that diffusive simulations well reproduce the experimental trends for short-legged plasmas, regardless the assumption of constant or radius-dependent perpendicular transport coefficients. This is the case for both a simple Monte Carlo particle tracer (MONALISA) and a more complex code simulating ions, electrons and neutrals (SolEdge2D-EIRENE). Adding a poloidal dependence (ballooning) of perpendicular transport coefficients to account for localized enhanced transport at the outer midplane, changes the symmetry of target profiles 
which exhibit a lower $S_{q} / \lambda_{q}$ ratio, close to what is observed experimentally. Simulations under these assumptions nevertheless yield a constant $\lambda_{q}$ and an increasing $S_{q}$. However, the increase in $\lambda_{n_{\mathrm{e}}}$ with $L_{\mathrm{div}}$ measured in the experiment is in qualitative agreement with the results of a first principle turbulent model (TOKAM3X) highlighting the importance of turbulent transport not only around the outer midplane but also in the vicinity of the X-point and all along the common flux region, causing a stronger broadening of the SOL in the case of a longer divertor leg. These results question the current interpretation of $\lambda_{q}$ as a purely main SOL quantity with $B_{\mathrm{P} \text {,omp }}$ as the only dependence on control parameters. In fact, if one assumes a symmetric spreading in the divertor region, in case of long leg configurations as the ones presented here, $\lambda_{q}$ appears to be related to transport in both main SOL and divertor SOL. The implications of this work on future machines like ITER and DEMO are nontrivial and difficult to test on other existing devices because of the limited flexibility of the magnetic geometry. If what ultimately matters for the enhancement of turbulent transport in the divertor is the ratio of the divertor leg length to the total parallel connection length, then a strong effect should not be expected. ITER (and possibly DEMO) will have small $L_{\text {div }} / L_{\|}$, similarly to most existing devices, and therefore could be satisfactorily described by diffusive codes, consistently with the short-legged TCV equilibria. If, on the other hand, the importance of turbulence in the divertor is inherently related to the length of its leg, then an extra spreading of heat flux profiles could in principle occur in future bigger devices, even in the case of traditional magnetic configurations.

\section{Acknowledgments}

This work has been carried out within the framework of the EUROfusion Consortium and has received funding from the Euratom research and training programme 2014-2018 under grant agreement No. 633053. The views and opinions expressed herein do not necessarily reflect those of the European Commission. This work was supported by the US Department of Energy under Grant No. DE-SC0010529.

\section{ORCID iDs}

A Gallo (ib https://orcid.org/0000-0002-7472-7830

H Reimerdes (1D https://orcid.org/0000-0002-9726-1519

C Theiler (i) https://orcid.org/0000-0003-3926-1374

C K Tsui (ii) https://orcid.org/0000-0002-7346-8312

\section{References}

[1] Loarte A et al 2007 Chapter 4: power and particle control Nucl. Fusion 47 S203

[2] Gunn J P, Carpentier-Chouchana S, Dejarnac R, Escourbiac F, Hirai T, Komm M, Kukushkin A, Panayotis S and Pitts R A
2016 Ion orbit modelling of ELM heat loads on ITER divertor vertical targets Nucl. Mater. Energy (https://doi. org/10.1016/j.nme.2016.10.005)

[3] Gunn J P et al 2017 Surface heat loads on the ITER divertor vertical targets Nucl. Fusion 57046025

[4] Pitts R A et al 2017 Physics conclusions in support of \{ITER W divertor monoblock shaping Nucl. Mater. Energy (https://doi.org/10.1016/j.nme.2017.03.005)

[5] Krasheninnikov S I, Kukushkin A S and Pshenov A A 2016 Divertor plasma detachment Phys. Plasmas 23055602

[6] Theiler C et al 2017 Results from recent detachment experiments in alternative divertor configurations on TCV Nucl. Fusion 57072008

[7] Stangeby P C 2000 The Plasma Boundary of Magnetic Fusion Devices (Bristol: IOP Publishing)

[8] Loarte A et al 1999 Multi-machine scaling of the divertor peak heat flux and width for L-mode and H-mode discharges J. Nucl. Mater. 266-269 587-92

[9] Wagner F 1985 A study of the perpendicular particle transport properties in the scrape-off layer of ASDEX Nucl. Fusion 25525

[10] Eich T, Sieglin B, Scarabosio A, Fundamenski W, Goldston R J and Herrmann A 2011 Inter-ELM power decay length for JET and ASDEX upgrade: measurement and comparison with heuristic drift-based model Phys. Rev. Lett. 107215001

[11] Makowski M A et al 2012 Analysis of a multi-machine database on divertor heat fluxes Phys. Plasmas 19056122

[12] Eich T et al 2013 Scaling of the tokamak near the scrape-off layer H-mode power width and implications for ITER Nucl. Fusion 53093031

[13] Scarabosio A, Eich T, Herrmann A and Sieglin B 2013 Outer target heat fluxes and power decay length scaling in L-mode plasmas at JET and AUG J. Nucl. Mater. 438 S426-30 Proc. 20th Int. Conf. on Plasma-Surface Interactions in Controlled Fusion Devices

[14] Fedorczak N, Gunn J P, Nace N, Gallo A, Baudoin C, Bufferand H, Ciraolo G, Eich T, Ghendrih P and Tamain P 2017 Width of turbulent SOL in circular plasmas: a theoretical model validated on experiments in tore supra tokamak Nucl. Mater. Energy (https://doi.org/10.1016/j. nme.2017.03.032)

[15] Militello F, Fundamenski W, Naulin V and Nielsen A H 2012 Simulations of edge and scrape off layer turbulence in mega ampere spherical tokamak plasmas Plasma Phys. Control. Fusion 54095011

[16] Horacek J et al 2016 Multi-machine scaling of the main SOL parallel heat flux width in tokamak limiter plasmas Plasma Phys. Control. Fusion 58074005

[17] Gunn J P et al 2007 Evidence for a poloidally localized enhancement of radial transport in the scrape-off layer of the tore supra tokamak J. Nucl. Mater. 363-365 484-90 Plasma-Surface Interactions-17

[18] Sieglin B, Eich T, Faitsch M, Herrmann A and Scarabosio A 2016 Investigation of scrape-off layer and divertor heat transport in ASDEX upgrade L-mode Plasma Phys. Control. Fusion 58055015

[19] Maurizio R et al Nucl. Fusion submitted

[20] Gallo A, Fedorczak N, Maurizio R, Theiler C, Elmore S, Labit B, Reimerdes H, Nespoli F, Ghendrih P and Eich T 2016 Effect of plasma geometry on divertor heat flux spreading: Monalisa simulations and experimental results from TCV Nucl. Mater. Energy (https://doi.org/10.1016/j. nme.2016.10.003)

[21] Bufferand $\mathrm{H}$ et al 2015 Numerical modelling for divertor design of the west device with a focus on plasmawall interactions Nucl. Fusion $\mathbf{5 5} 053025$

[22] Tamain P, Bufferand H, Ciraolo G, Colin C, Galassi D, Ghendrih P, Schwander F and Serre E 2016 The 
TOKAM3X code for edge turbulence fluid simulations of tokamak plasmas in versatile magnetic geometries

J. Comput. Phys. 321 606-23

[23] Galassi D, Tamain P, Bufferand H, Ciraolo G, Ghendrih P, Baudoin C, Colin C, Fedorczak N, Nace N and Serre E 2017 Drive of parallel flows by turbulence and large-scale $E \times B$ transverse transport in divertor geometry Nucl. Fusion $\mathbf{5 7}$ 036029

[24] Behn R, Alfier A, Medvedev S Y, Zhuang G, Pasqualotto R, Nielsen P and Martin Y 2007 Edge profiles of electron temperature and density during ELMy H-mode in ohmically heated TCV plasmas Plasma Phys. Control. Fusion 491289

[25] Boedo J A, Crocker N, Chousal L, Hernandez R, Chalfant J, Kugel H, Roney P and Wertenbaker J 2009 Fast scanning probe for the NSTX spherical tokamak Rev. Sci. Instrum. 80 123506
[26] Pitts R A, Alberti S, Blanchard P, Horacek J, Reimerdes H and Stangeby P C 2003 ELM driven divertor target currents on TCV Nucl. Fusion 431145

[27] Greenwald M 2002 Density limits in toroidal plasmas Plasma Phys. Control. Fusion 44 R27

[28] Canal G P, Lunt T, Reimerdes H, Duval B P, Labit B and Vijvers W A J 2015 Enhanced exb drift effects in the TCV snowflake divertor Nucl. Fusion 55123023

[29] Carralero D, Birkenmeier G, Mller H W, Manz P, deMarne P, Mller S H, Reimold F, Stroth U, Wischmeier M and Wolfrum E 2014 An experimental investigation of the high density transition of the scrape-off layer transport in ASDEX upgrade Nucl. Fusion 54123005

[30] LaBombard B et al 2004 Transport-driven scrape-off-layer flows and the boundary conditions imposed at the magnetic separatrix in a tokamak plasma Nucl. Fusion 441047 